\title{
Honorararzt im Krankenhaus - Arbeitnehmer oder selbstständige Tätigkeit?
}

\author{
- Anmerkung zum Urteil des Landesarbeitsgerichts Hessen \\ vom 14.01.2013 - Az.: 16 Sa 1213/12) -
}

\section{Einführung \\ $\nabla$}

Die Frage der Arbeitnehmereigenschaft von Honorarärzten in Krankenhäusern ist auf Grund der damit verbundenen nicht nur arbeitsrechtlichen, sondern auch sozialversicherungsrechtlichen sowie steuerrechtlichen Konsequenzen in der Praxis von großer Bedeutung. So können sich aus der arbeitsrechtlichen Feststellung der Arbeitnehmereigenschaft eines Honorararztes Rechtsfolgen wie die Anwendung des Kündigungsschutzgesetzes, des Bundesurlaubsgesetzes oder von Tarifverträgen ergeben. Bei einer Einstufung der honorarärztlichen Tätigkeit als sozialversicherungspflichtig können Beiträge zur Arbeitslosen-, Kranken, Renten und Pflegeversicherung, sowie zur Unfallversicherung anfallen. Steuerrechtlich gesehen stellt sich die Frage der Abführung von Lohnsteuer.

Mit der aktuellen Neuregelung des $\S 2$ Abs. 1 KHEntgG, durch die nunmehr festgeschrieben wird, dass Krankenhausleistungen auch durch nicht am Krankenhaus angestellte Ärzte erbracht werden können, hat der Gesetzgeber auf die divergierende Rechtsprechung der Sozialgerichtsbarkeit und der Verwaltungsgerichtsbarkeit reagiert und klargestellt, dass grundsätzlich die Erbringung und Vergütung von allgemeinen Krankenhausleistungen nicht vom Status des ärztlichen Personals im Krankenhaus abhängt (vgl. Gesetzesbegründung, BT-Drs. 17/9992, S. 26). Auch wenn nach dieser gesetzgeberischen Tätigkeit die Frage der Arbeitnehmereigenschaft des Honorararztes unter krankenhausrechtlichen Gesichtspunkten keine entscheidende Rolle mehr spielen mag, so wird sich die arbeitsgerichtliche sowie sozialgerichtliche Rechtsprechung auch zukünftig weiterhin mit der Problemstellung auseinandersetzen müssen.

Ein aktuelles Beispiel ist das Urteil des Landesarbeitsgerichts (LAG) Hessen vom 14.01.2013 - Az.: 16 Sa 1213/12, welches in dem konkret zu entscheidenden Einzelfall die Arbeitnehmereigenschaft eines als Honorararzt in einem Krankenhaus tätigen Radiologen verneint hat. Anhand der
Entscheidungsgründe des LAG sollen nachfolgend die aus arbeitsrechtlicher Sicht für die Beurteilung der Arbeitnehmereigenschaft wesentlichen Merkmale herausgearbeitet werden.

\section{Arbeitnehmerbegriff des BAG $\nabla$}

Ausgangspunkt der rechtlichen Überlegungen ist der durch die ständige Rechtsprechung des Bundesarbeitsgerichts (BAG) entwickelte Arbeitnehmerbegriff, wonach Arbeitnehmer im arbeitsrechtlichen Sinn derjenige ist, der aufgrund eines privatrechtlichen Vertrages im Dienst eines anderen zur Leistung weisungsgebundener fremdbestimmter Arbeit in persönlicher Abhängigkeit verpflichtet ist. Wie das LAG Hessen in seinen Entscheidungsgründen ausführt, ist die geschuldete Leistung dabei im Rahmen einer von Dritten bestimmten Arbeitsorganisation zu erbringen.

Die Einordnung als Arbeitnehmer werde, so das LAG Hessen, insbesondere dadurch geprägt, dass der Beschäftigte dem Weisungsrecht seines Vertragspartners (des Arbeitgebers) hinsichtlich Inhalt, Durchführung, Zeit, Dauer und Ort der Tätigkeit unterliegt. Ein Arbeitnehmer könne seine Tätigkeit und Arbeitszeit nicht frei gestalten, was demgegenüber jedoch wesentliches Charakteristikum des Selbstständigen sei.

Wie das LAG Hessen noch einmal hervorhebt, kommt es für die Frage, ob Selbstständigkeit oder eine abhängige Beschäftigung vorliegt, nicht auf den Wortlaut der vertraglichen Vereinbarung, sondern allein auf den tatsächlich gelebten Geschäftsinhalt an.

\section{Tätigkeit in Räumlichkeiten des Krankenhauses kein Indiz für Arbeitsverhältnis $\nabla$}

Bezogen auf den konkret zu entscheidenden Fall sprach nach Ansicht des LAG Hessen die Tatsache, dass der Honorararzt sei-

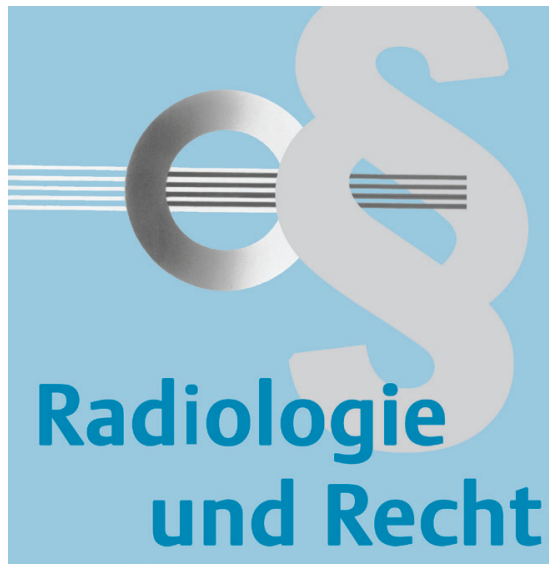

ne Tätigkeit örtlich gesehen nur im Krankenhaus ausüben konnte, nicht zwingend für die Annahme einer Arbeitnehmereigenschaft. Dies entspreche der Tätigkeit eines freiberuflichen Belegarztes, der auch die Räumlichkeiten eines Krankenhauses nutze und mit den dortigen Mitarbeitern zusammenarbeite. Ebenso sei es unbeachtlich, dass die Tätigkeit des Honorararztes zuvor durch einen angestellten Arzt ausgeübt wurde.

\section{Tageshonorar indiziert kein zeitliches Direktionsrecht $\nabla$}

Darüber hinaus weist das LAG Hessen in seiner Entscheidung darauf hin, dass ein vereinbartes Tageshonorar (im vorliegenden Fall 1.000 Euro) kein Direktionsrecht in zeitlicher Hinsicht bedeute. Dies gelte insbesondere auch dann nicht, wenn verbunden mit dem Tageshonorar ein Zeitfenster für die Tätigkeit, beispielsweise „max. 8 bis 16:00 Uhr, für Überstunden der durchschnittliche Stundensatz“, vereinbart wurde. Dadurch würde nicht die zeitliche Lage der Arbeitszeit verbindlich festgeschrieben, sondern vielmehr nur geregelt, für welche Tätigkeitsdauer das Tageshonorar anfallen solle. Selbst bei der Annahme einer Regelung der täglichen Dienstzeit, handele es sich dabei nicht um ein einseitiges Direktionsrecht des Arbeitnehmers, sondern um eine entsprechende einvernehmliche vertragliche Vereinbarung.

\section{Bemühung um Zuweiserstrukturen $\nabla$}

In dem vom LAG Hessen zu entscheidenden Fall oblag es dem Honorararzt u.a., die Zuweiserstrukturen des Krankenhauses auszubauen. Dazu hatte der Radiologe Kontakt zu möglichen zuweisenden Ärz- 
ten aufgenommen, sich ihnen vorgestellt und um Zuweisung von Patienten gebeten. Das LAG Hessen wertet diese Tätigkeit nicht als eine Stabsfunktion, die nur von Arbeitnehmern des Krankenhauses wahrgenommen werden könne.

\section{Unbefristeter Honorararztvertrag möglich}

Schließlich sieht das LAG Hessen zudem kein Argument für ein Arbeitsverhältnis darin, wenn die Vereinbarung zwischen Honorararzt und Krankenhaus unbefristet abgeschlossen wird. Durch eine unbefristete Gestaltung ergebe sich kein zwingender Hinweis für eine persönliche Abhängigkeit des Honorararztes.

\section{Fazit}

$\nabla$

Das dargestellte Urteil des LAG Hessen zeigt mögliche Gestaltungsvarianten im Rahmen von Honorararztvereinbarungen auf, die aus arbeitsrechtlicher Sicht nicht zur Annahme einer Scheinselbstständigkeit und damit eines Arbeitsverhältnisses bei honorarärztlichen Strukturen führen. So spreche insbesondere ein an bestimmte Arbeitszeiten geknüpftes Tageshonorar, die unbefristete Vertragsgestaltung und die Aufgabe des Aufbaus von Zuweiserstrukturen nicht zwingend für eine Arbeitnehmereigenschaft des Honorararztes.

Wie bereits einleitend erwähnt, ist jedoch bei der statusrechtlichen Einordnung von Honorarärzten neben der arbeitsrechtlichen Betrachtung auch das Sozialversicherungsrecht zu beachten. Dabei sind die Sozialgerichte bei der Prüfung sozial- rechtlicher Fragestellungen nicht an die arbeitsrechtliche Beurteilung der Arbeitnehmereigenschaft gebunden, so dass voneinander abweichende Beurteilungen der beiden Gerichtsbarkeiten im Hinblick auf die Statusfrage von Honorarärzten denkbar sind. Unter sozialversicherungsrechtlichen Gesichtspunkten hängt die Statusentscheidung vorrangig davon ab, inwiefern der Honorararzt in die fremde Arbeitsorganisation eingebunden ist und inwieweit der Honorararzt selbst ein nennenswertes unternehmerisches Risiko trägt. So sehen die Sozialgerichte z.B. eine Vergütung auf Stundenbasis, die Aufnahme des Honorararztes in Dienstpläne oder die regelmäßige Tätigkeit an bestimmten Tagen in der Woche als Indiz für eine abhängige Beschäftigung an (vgl. SG Münster, Urt. v. 18.12.2012 - Az.: S 14 R 180/12).

Zur Vollständigkeit sei an dieser Stelle auf ein aktuelles Urteil des Landessozialgerichts (LSG) Stuttgart vom 17.04.2013 (Az.: L 5 R 3755/11) hingewiesen, in welchem das LSG Stuttgart nicht nur die grundsätzliche Zulässigkeit der honorarärztlichen Tätigkeit im Krankenhaus in Frage stellt, sondern darüber hinaus zumindest die Niederlassung des Arztes als zwingende Voraussetzung für die selbstständige Tätigkeit als Honorararzt im Krankenhaus fordert. Nach Ansicht des LSG Stuttgart beziehe sich der neue Wortlaut des $\S 2$ Abs. 1 KHEntgG, wonach Krankenhausleistungen auch Behandlungen durch nicht fest angestellte Ärztinnen und Ärzte sind, allein auf Ärzte im zeitlich befristeten Arbeitsverhältnis und nicht auf gar nicht angestellte bzw. niedergelassene Ärzte. Diese Auslegungsweise widerspricht jedoch dem bereits einleitend erwähnten Willen des Gesetzgebers und dem eindeutigen Wortlaut der Gesetzes- begründung. Es bleibt abzuwarten, inwiefern dieses Urteil des LSG Stuttgart einer Überprüfung im Revisionsverfahren vor dem Bundessozialgericht (BSG) Stand hält.

Die aktuelle arbeitsgerichtliche sowie sozialgerichtliche Rechtsprechung machen deutlich, dass trotz der jüngsten gesetzgeberischen Entwicklungen die honorarärztliche Tätigkeit im Krankenhaus weiterhin eine Vielzahl von rechtlichen Fragestellungen aufwirft, die auch in Zukunft weiterhin einzelfallabhängig zu beurteilen sein werden. Im Rahmen der vertraglichen Ausgestaltung einer selbstständigen honorarärztlichen Tätigkeit sollte darauf geachtet werden, dass der Honorararzt nach dem individuellen Bedarf und nicht regelmäßig wiederkehrend beauftragt wird. Insbesondere sollten regelmäßige Beauftragungen nicht in einem Rahmenvertrag festgehalten werden.

\section{Urs Frigger, Rechtsanwalt}

\section{Dr. Ulrike Tonner, Rechtsanwältin}

Rechtsanwälte Wigge

Scharnhorststr. 40

48151 Münster

Telefon: (0251) 53595-0

Telefax: (0251) 53595-99

Internet: www.ra-wigge.de

E-Mail: kanzlei@ra-wigge.de 